\title{
Carpal Tunnel Syndrome as an Unusual Immune Checkpoint Inhibitor Adverse Effect: A Case Series and Review of the Literature
}

\author{
Walid Shalata "a, Alexander Yakobson " a, Ismaell Massalha a , Roxana D. Grinberg a , Iris M. Goldstein ${ }^{\text {b }}$ \\ Rachel Gibbs ${ }^{c}$, Roy Grinberg ${ }^{d}$, Ala Eddin Neime ${ }^{\mathrm{e}}$, Ali Abu-Juma'a ${ }^{\mathrm{e}}$, Waleed Kian ${ }^{\mathrm{a}}$, Nir Peled ${ }^{\text {a }}$, Keren Rouvinov ${ }^{\mathrm{a}}$ \\ ${ }^{a}$ The Legacy Heritage Oncology Center \& Dr. Larry Norton Institute, Soroka Medical Center \& Ben-Gurion University, \\ Beer-Sheva, Israel. \\ ${ }^{\mathrm{b}}$ Department of Neurology, Soroka University Medical Center, Faculty of Health Sciences, Ben Gurion University, \\ Beer Sheva, Israel. \\ ${ }^{c}$ Medical School for International Health and Sciences, Ben-Gurion University, Beer Sheva, Israel. \\ ${ }^{\mathrm{d}}$ Department of Plastic Surgery, Soroka University Medical Center, Faculty of Health Sciences, Ben Gurion University, \\ Beer Sheva, Israel. \\ e Department of Internal Medicine, Soroka University Medical Center, Faculty of Health Sciences, Ben Gurion University, \\ Beer Sheva, Israel.
}

*These two authors contributed equally to this work.

*Corresponding Author: Dr. Walid Shalata, MD, Resident Physician in Oncology, The Legacy Heritage Oncology Center \& Dr. Larry Norton Institute, Soroka Medical Center \& Ben-Gurion University, Beer-Sheva, Israel; walid.shalata@ gmail.com

\begin{abstract}
While immune checkpoint inhibitors (ICIs) have transformed standards of care and drastically improved patient prognoses in several malignant diseases, a range of immune related adverse events (irAEs) from ICIs have been observed. This case series describes the clinical course of three patients with unusual immune checkpoint-inhibitor (ICI) induced toxicities. These three patients developed carpal tunnel syndrome (CTS) while on ICI therapy. The first patient was a 79 year-old male who received neo-adjuvant chemo-immunotherapy and adjuvant immunotherapy (atezolizumab) for stage II-B lung adenocarcinoma, the second patient was a 70 year-old female who received immunotherapy only (nivolumab) for stage IV renal cell carcinoma and the third patient was a 71 year-old male who received adjuvant immunotherapy (nivolumab) for stage 2-A melanoma. The patients developed carpal tunnel syndrome with severe neuropathy as a result of therapy. All patients had complete symptomatic relief when treated with either corticosteroids or intravenous immunoglobulins for CTS.
\end{abstract}

Keywords: Atezolizumab - Nivolumab - Carpal tunnel syndrome - Lung Carcinoma - Renal Cell Carcinoma - Immune Checkpoint Inhibitors (ICIs) - Immune Related Adverse Events (irAEs)

\section{Introduction}

Immune checkpoint inhibitors (ICIs) have transformed the prognosis of several malignancies establishing new standards of care in adjuvant, neo-adjuvant and metastatic settings. Nivolumab and atezolizumab are humanized IgG4 monoclonal antibodies targeted against major immune checkpoint receptors regulating $\mathrm{T}$ cell response. Nivolumab is directed against the programmed cell death receptor 1 (PD-1) while atezolizumab is targeted against programmed cell death-ligand 1 (PD-L1). By blocking PD-1 and PD-L1 activity these ICI's enhance anti-tumor T-cell activity. However, improved T-cell proliferation may also lead to irAEs ${ }^{[1,2]}$.

An analysis of 3953 patients from multiple clinical trials revealed that the most frequent irAEs include rash, diarrhea, nausea, myalgia, arthralgia, and pneumonitis. Aside from rash, cutaneous adverse effects included pruritus and less commonly vitiligo ${ }^{[3]}$. Neurologic adverse events from immune checkpoint 
inhibition have also been increasingly recognized despite the presenting symptoms and signs often being sub-acute and highly variable $^{[4]}$. Neurologic irAEs may be relatively less common than other irAEs, though the potential for long-term morbidity and even mortality is substantial ${ }^{[5]}$. Carpal tunnel syndrome (CTS) is the most common nerve entrapment syndrome in the upper extremity with a prevalence of 50 cases per 1000 individuals per year in the United States and more cases in women than in men ${ }^{[6]}$. In most CTS cases, an underlying etiology cannot be identified. Systemic conditions, such as renal failure, rheumatoid arthritis, and diabetes mellitus may also predispose patients to CTS. Patients with CTS often present with nocturnal pain, numbness, as well as tingling in the thumb and radial fingers bilaterally. Paresthesias can radiate proximally along the median nerve to the forearm and may even involve the ulnar digits ${ }^{[7]}$. Since CTS remains a clinical diagnosis, the role of electrodiagnostic and imaging studies is considered complementary.

To the best of our knowledge, CTS has not been previously reported in association with anti PD-L1 or anti PD-1 treatment.

\section{Case No .1}

A 79-year old male was referred to the emergency room in March 2019 by a primary care physician due to hemoptysis of one month's duration. He had experienced right sided chest pain for the previous three months and reported a $4 \mathrm{~kg}$ weight loss over the past month. The patient had a history of benign prostatic hyperplasia, was a smoker (30 pack-years (PY), and had been on treatment with a beta blocker, angiotensin-converting-enzyme inhibitor and aspirin since suffering from a myocardial infarction 10 years previously. He had a family history of a sister with renal cell carcinoma diagnosed at age 79 .

Physical examination, including lung and cardiovascular evaluation was normal and electrocardiogram was unremarkable. Routine laboratory investigations (complete blood count and biochemical profile) showed no abnormalities. Chest computed tomography (CT) showed an obstructing endo-bronchial mass $(7 \times 3.5 \mathrm{~cm})$ in the right lower lung (RLL). Bronchoscopy was performed with biopsy of the RLL mass. Pathological result showed- squamous cell carcinoma, moderately differentiated, of lung origin. Magnetic resonance imaging (MRI) of the head showed no evidence of metastatic disease and positron emission tomography-computed tomography (PET-CT) showed hyper- metabolic uptake in the RLL mass and in the right mediastinal area. The presumptive clinical diagnosis was stage T3 N0 M0 (stage 2B) non-small cell lung cancer.

A multidisciplinary conference including an oncologist, cardiothoracic surgeon and radiologist recommended systemic neoadjuvant therapy. The patient was enrolled in a neo-adjuvant treatment trial and received carboplatin (dosed to area under the curve (AUC)-5), atezolizumab (at a dose of $1200 \mathrm{mg}$ ) on day 1 every 21 days and nab-paclitaxel (Abraxane $\left.{ }^{\mathrm{TM}}\right)\left(80 \mathrm{mg} / \mathrm{m}^{2}\right)$ on days 1,8 , and 15 .

After receiving the second cycle the patient began to experience pain in the wrists bilaterally but more severe on the right side. After the third cycle he was re-admitted for chest CT that demonstrated a very good response with reduction of the mass to $1.1 \mathrm{~cm}$ in greatest diameter. In August 2019 the patient underwent tumor resection (right lower lobectomy), with pathologic margins free of disease.

Post-operatively the patient continued treatment with atezolizumab alone (fixed dose of $1200 \mathrm{mg}$ ) on day one every 21 days. After four cycles of treatment, the patient complained of intense pain with mild edema in both wrists, but again more severe on the right side. The treatment was stopped and re-evaluation with CT of head and chest showed no obvious reasons for his clinical symptoms. He was referred for neurologic and neurosurgical consultations. Both consultants noted that the patient was suffering from bilateral median and ulnar nerve palsies, with the rest of the neurologic examination described as normal. The suspected cause was severe CTS. An EMG was performed which showed prolonged distal sensory and motor latency, low snaps, borderline compound muscle action potential (CMAP) test and $\mathrm{f}$ waves of the right median nerve, as well as prolonged distal sensory and latency of the left median nerve. EMG findings were consistent with severe right CTS and mild left CTS (Table 1 and Table 2). He was then referred to an orthopedic surgeon and underwent open surgery of the right hand with bisection of the carpal tunnel ligament to free the nerve. For several weeks after surgery, the neuropathy and pain continued to progress with exacerbation of muscle weakness in the median and ulnar distribution.

At that point the patient was admitted for treatment with intravenous immunoglobulins (IV-IG) $(0.4 \mathrm{mg} / \mathrm{kg}$ daily for 5 days). By the third day of treatment a clinical improvement in both hands was noted with a return to normal motor and sensory function (Figure 1).

Table 1: Results of the nerve conduction velocity (NCV).

\begin{tabular}{|l|l|c|c|c|c|c|c|c|c|c|c|}
\hline Motor Nerves & & Lat $(\mathbf{m s})$ & SD & Amp $(\mathbf{m V})$ & SD & CV $(\mathbf{m} / \mathbf{s})$ & SD & Amp \% & SD & F-M (ms) & SD \\
\hline Right Median & Wrist-APB & 6 & 6.7 & 5.4 & -0.6 & 51 & -0.4 & -20 & -1.1 & 29.2 & 6.6 \\
\hline & Ab Elb-Wrist & 10.9 & & 4.3 & & & & & & \\
\hline Left & Wrist-APB & 3.9 & 0.5 & 9.4 & 0.8 & 51 & -0.4 & 1 & 0.7 & 25.1 & 2.8 \\
\hline & Ab Elb-Wrist & 8.4 & & 9.5 & & & & & & & \\
\hline Right Ulnar & wrist-ADM & 2.9 & -0.5 & 10.4 & -0.3 & 57.1 & 0.4 & -12 & 0.3 & 28.5 & 5.3 \\
\hline & Ab Elb-Wrist & 7.8 & & 9.1 & & & & & & & \\
\hline Left Ulnar & Wrist- ADM & 3 & -0.2 & 9.8 & -0.5 & 60 & 1 & & 1.8 & 28.3 & 5.1 \\
\hline & Ab Elb-wrist & 7.5 & & 9.9 & & & & & & & \\
\hline
\end{tabular}

Table 2: Results of EMG.

\begin{tabular}{|c|c|c|c|c|}
\hline Motor Nerves & & Lat (ms) & SD & $\operatorname{Amp}(\mathbf{m V})$ \\
\hline Right Median & Palm - Wrist & 7.8 & 34 & 4.4 \\
\hline Left Median & Palm - Wrist & 3.8 & 11.1 & 19 \\
\hline Right Ulnar & Dig V - Wrist & 3 & 0.7 & 21 \\
\hline Left Ulnar & Dig V - Wrist & 3.5 & 2 & 26 \\
\hline
\end{tabular}




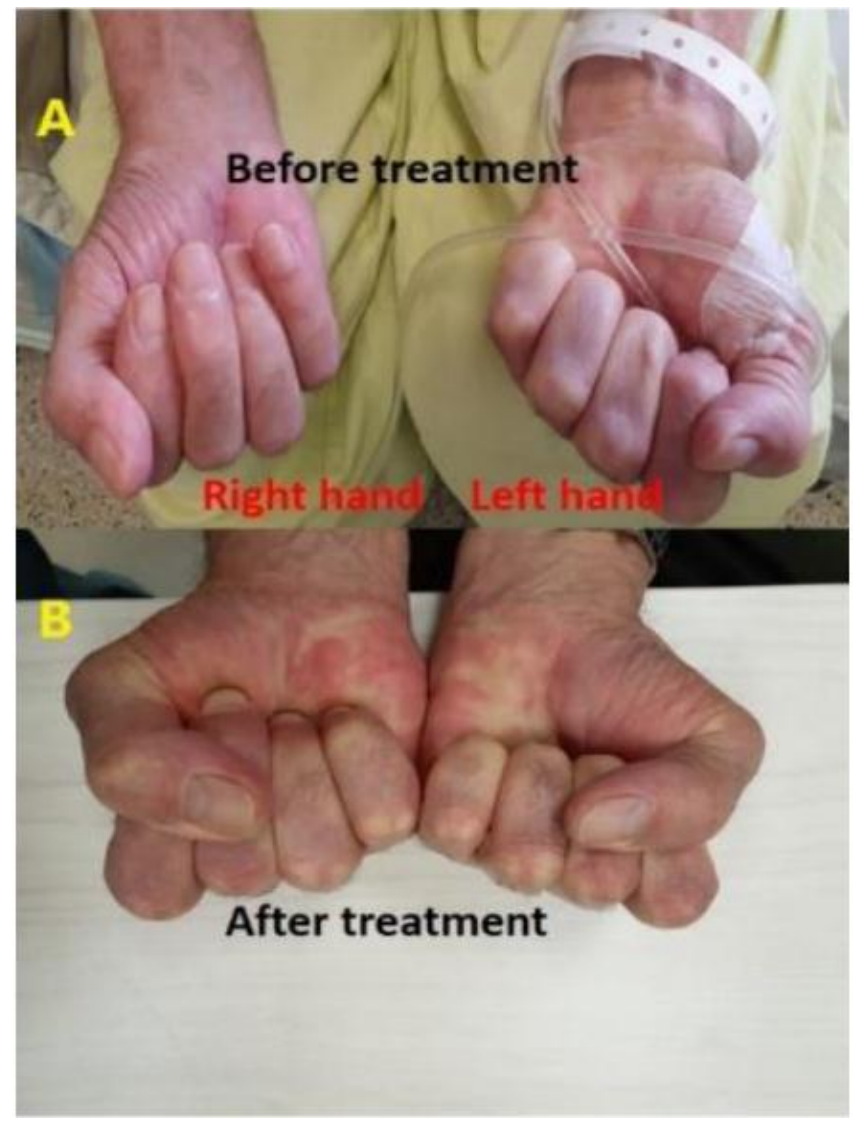

Figure 1: Difference in difficulty of closing palms and maintaining hand-grip before (A) and after (B) IV-IG treatment.

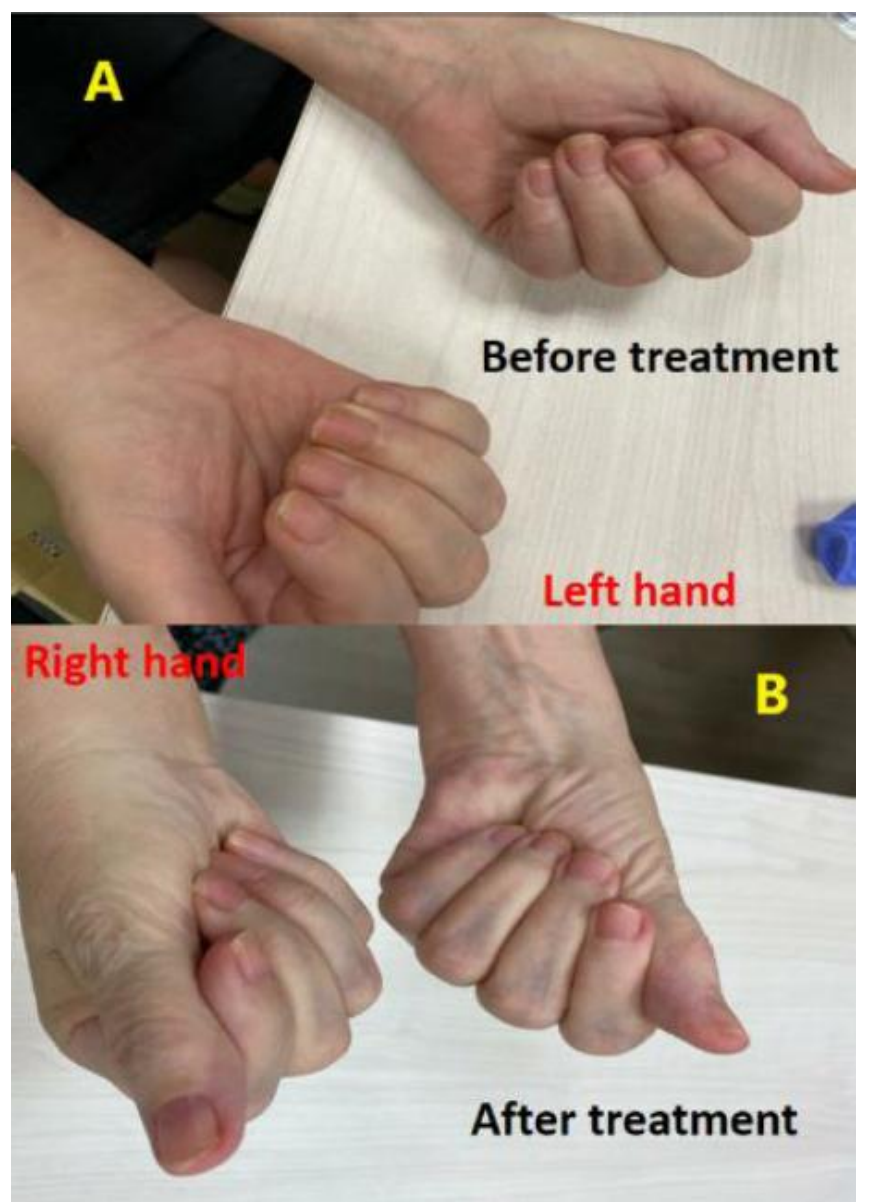

Figure 2: Difference in difficulty of closing palms and maintaining hand-grip before (A) and after (B) corticosteroid treatment.

\section{Case No .2}

A 70 rear-old female with no significant medical or family history was referred to the emergency room in May 2015 by a primary care physician due to increasing left flank pain and discomfort over the past two months.

The patient underwent CT of the abdomen, which showed a mass on the left kidney with involvement of the left renal vein and lymphadenopathy. For further investigation she had a PET-CT that showed the same results as in the $\mathrm{CT}$ of the abdomen with no other distant metastasis. In July, 2015 she underwent left radical nephrectomy, and histopathologic findings showed renal cell carcinoma (RCC), clear cell, involving the renal vein along with 5 of 8 lymph nodes. The pathologic stage was determined to be T3 N1 M0 (stage 3). The patient refused any type of treatment or follow up. In April 2016 she presented with extradural and intracranial metastases. She was admitted for an excisional biopsy of the extradural metastasis. Histopathologic findings revealed metastatic RCC. PET-CT showed hyper-metabolic uptake in the left lung helium $(17 \mathrm{~mm})$ and left mediastinal area, as well as in peripheral nodules in both lungs $(6 \mathrm{~mm})$, proximal left humerus, right femoral neck and left adrenal (stage TX N0 M1 stage 4). Pazopanib therapy was initiated ( $800 \mathrm{mg}$ orally once daily). At follow-up in October 2017 a total body CT showed disease progression in the lung and mediastinum.

Nivolumab $(3 \mathrm{mg} / \mathrm{kg})$ was started with good radiological response. In March 2020 the patient complained of intense pain in both wrists with increased severity on the right side. A multidisciplinary conference including an oncologist and immunologist came to the conclusion that the patient had CTS secondary to ICI therapy. Nivolumab was stopped and the patient was referred for neurologic consultation.

Neurologic examination showed that the patient was suffering from bilateral median and ulnar nerves palsies. Due to the suspicion of CTS induced by nivolumab, corticosteroid therapy was initiated with prednisone $(1 \mathrm{mg} / \mathrm{kg})$. After one week with minor improvement, the dose was raised to $2 \mathrm{mg} / \mathrm{kg}$. After an additional two weeks of treatment the patient reported a significant reduction in pain. Follow-up neurologic exam showed improvement in the ulnar and medial nerve palsies (Figure 2).

\section{Case No .3}

A 71-year old male with a history of melanoma in 2012 (stage 1, resected). At follow-up with a dermatologist in February 2019 he was noted to have two new skin lesions that appeared to be sebaceous cysts $(4 * 2 \mathrm{~cm}$ and $2 * 2 \mathrm{~cm}$ in diameter) in the left upper back. He was referred for PET-CT which showed hyper-metabolic uptake in the left upper back.

A multidisciplinary conference including an oncologist, plastic-surgeons and a radiologist came to the conclusion that the findings represented recurrence of melanoma and recommended wide excision of the area.

The patient underwent wide excision with histopathologic findings showing metastatic melanoma. The pathologic stage was determined to be T3A N0 M0 (stage 2-A). Molecular testing showed BRAF wild type. The patient received systemic intravenous immunotherapy consisting of nivolumab $3 \mathrm{mg} / \mathrm{kg}$ on day one every 2 weeks for 12 cycles (during this period PET-CT showed no evidence of disease). Due to the COVID-19 pandemic the dose of nivolumab was escalated to $6 \mathrm{mg} / \mathrm{kg}$ on day one to enable treatment on an every 4 week basis. After two cycles ( 8 weeks) of treatment on the higher dose the patient experienced 
intense pain in both wrists, with increasing pain and weakness after the third cycle (12 weeks).

Based on the experience with the previous 2 cases described above, nivolumab was stopped with the suspected diagnosis of carpal tunnel syndrome and the patient was referred for orthopedic surgeon and neurologic consultations. Neurologic examination showed that the patient was suffering from bilateral median and ulnar nerve neuropathy. Imaging with plain X-ray and ultrasound of the wrists showed fluid collection between the mildly swollen flexor tendons diagnostic of tendovaginitis (tenosynovitis, trigger-finger).

Corticosteroid therapy was then initiated with prednisone $(1.5 \mathrm{mg} / \mathrm{kg})$. After 10 days the patient reported a significant improvement and reduction in pain. Later in July 2020, the patient underwent a total body CT for follow-up which showed no evidence of disease.

\section{Discussion and Conclusion}

Recognition and treatment of ICI neurological toxicities can be challenging. Immune-suppressant treatments, including IV and oral corticosteroids, immunoglobulin, as well as plasma exchange, can lead to partial recovery. IV IG is one of the treatments of choice for irAEs, but it is usually reserved for severe cases. The spectrum of neurological complications from ICIs therapy has also been expanded to include painful brachial plexus neuritis ${ }^{[8]}$. This case series supports the notion that unusual or rare adverse effects may be a predictive factor for response to ICI therapy ${ }^{[9]}$.

To the best of our knowledge, CTS associated with ICI therapy has not been reported. The rapid improvement of symptoms after steroids and IV IG treatment and the bilateral occurrence, suggest a neurologic immune adverse effect and not an independent synchronous occurrence. While the exact immunemediated mechanism is not clear, peripheral T-cell dysregulation seems likely.

Withholding ICIs and initiating high-dose corticosteroids has been beneficial in most neurological irAEs. Whether to add corticosteroid sparing agents in the course of treatment of the irAEs needs to be considered on an individual basis.

\section{Data Availability}

Not applicable

\section{Disclosure Statement}

The authors declare no conflict of interest.

\section{Funding Sources}

The authors received no financial support for the research, authorship, and/or publication of this article.

\section{Acknowledgements}

The authors thank David B. Geffen, MD for his critical review of the manuscript.

\section{Statement of Ethics}

The authors have no ethical conflicts to disclose.

\section{References}

[1] Postow MA, Sidlow R, Hellmann MD. Immune-related adverse events associated with immune checkpoint blockade. N Engl J Med. 2018;378(2):158-68.

[2] Spain L, Tippu Z, Larkin JM, et al. How we treat neurological toxicity from immune checkpoint inhibitors ESMO Open 2019;4:e000540. doi: 10.1136/esmoopen2019-000540

[3] Wang DY, Salem J-E, Cohen JV, et al. Fatal Toxic Effects Associated with Immune Checkpoint Inhibitors: A Systematic Review and Meta-analysis. JAMA Oncol2018; 4:1721-8.doi:10.1001/jamaoncol.2018.3923

[4] Bickel KD. Carpal tunnel syndrome. J Hand Surg Am. 2010;35:147-52

[5] Neligan C. Peter, Plastic surgery, Hand and Upper Extremity, James Chang, Fourth Edition, Vol. 6, Elsevier 2018, pag. 530

[6] Johnson, D.B., Manouchehri, A., Haugh, A.M. et al. Neurologic toxicity associated with immune checkpoint inhibitors: a pharmacovigilance study. j. immunotherapy cancer 7, 134 (2019). https://doi.org/10.1186/s40425019-0617-x

[7] Trinh S, Le A, Gowani S, La-Beck NM. Management of Immune-Related Adverse Events Associated with Immune Checkpoint Inhibitor Therapy: a Minireview of Current Clinical Guidelines. Asia Pac J Oncol Nurs. 2019;6(2):154-160. doi:10.4103/apjon.apjon_3_19

[8] Connie Jackaman, Christine S. Bundell, Beverley F. Kinnear, Alison M. Smith, Pierre Filion, Deborah van Hagen, Bruce W. S. Robinson and Delia J. NelsonJ Immunol November 15, 2003, 171 (10) 5051-5063; DOI: https://doi.org/10.4049/jimmunol.171.10.5051

[9] Shalata W, Peled N, Gabizon I, Abu Saleh O, Kian W, Yakobson A: Associated Myocarditis: A Predictive Factor for Response? Case Rep Oncol 2020;13:550-557. https://doi.org/10.1159/000507278 